Expressed constitutional disorders (fever, weight loss) observed in 13 pts (91.7\%). 11 pts $(78.6 \%)$ had acute cutaneous lupus, 5 pts $(35.7 \%)$ - chronic cutaneous lupus, $3(21.4 \%)$ - oral and nasal ulcers, $6(42.8 \%)$ - nonscarring alopecia, 9 pts $(64.3 \%)$ - polyarthritis, 2 pts $(14.3 \%)$ - renal involvement, 2 pts $(14.3 \%)$ - serositis, $1(7.1 \%)$ - interstitial lung disease, 5 pts $(35.7 \%)$ - neuropsychiatric disorder, including psychosis in 2 (14.3\%). 4 patients had skin lesions atypical for SLE (1 - annular erythema, 1 - erythema nodosum, 2 - Ro-associated skin vasculitis). 12 pts (85.7\%) had generalized lymphadenopathy. 12 pts $(85.7 \%)$ had various hematological disorders: anemia - in 5 pts (35.7\%), leukopenia - in 9 (64.3\%), isolated lymphopenia in $1(7.1 \%)$, thrombocytopenia - in 4 pts $(28.6 \%) .13$ pts had isolated involvement of salivary glands, $1-$ combined with lacrimal glands. The decrease in salivary gland function was recorded in $50 \%$ of cases, hypolacrimia - in one case. Recurrent parotitis was present in only one case (7.1\%). ANA were detected in $100 \%$ pts, anti-dsDNA - in 10 pts (71.4\%), anti-Sm - in 7 pts (50.0\%), anti-Ro - in $10(71.4 \%)$, anti-La - in 7 (50\%), RNP-70 - in 5 pts (35.7\%), RF+ - in 6 pts (42.9\%), hypocomplementemia - in 3 pts $(21.4 \%)$. The most common was the combination of positive ANA, anti-dsDNA, antiRo with acute cutaneous lupus, polyarthritis, generalized lymphadenopathy and expressed constitutional disorders -8 pts $(57.1 \%) .4$ pts $(28.6 \%)$ had polyclonal hypergammaglobulinemia. 3 pts $(21.4 \%)$ had concomitant autoimmune non-rheumatic disease; 1 - autoimmune hepatitis, 1 - type 1 diabetes mellitus, 1 - autoimmune thyroiditis. Median disease activity by SLEDAI at the time of jSLE verification was 11.5 scores [9.25;15.7]

Conclusion: According to our results, the frequency of detection of secondary SS in jSLE was higher than the literature data. The clinical features include a high frequency of constitutional disorders, lymphadenopathy, skin manifestations, high frequency of antiRo with a significantly lower incidence of kidney involvement, serositis than jSLE without SS. In pts with a diagnosis of SLE, the possibility of developing secondary SS should be considered (specially in girls with antiRo positive), the early detection of which affects the choice of therapy and prognosis. References: [1]Malagón C, Gomez M, Mosquera C et al. Juvenile polyautoimmunity in a rheumatology setting. Autoimmunity Reviews, Volume 18, Issue 4, 2019, p 369-381. https://doi.org/10.1016/j.autrev.2018.11.006.

Disclosure of Interests: None declared

DOI: 10.1136/annrheumdis-2020-eular.3271

\section{AB0996 FAMILY CASE OF BLAU SYNDROME IN 3 CHILDREN, INITIALLY DIAGNOSED AS JUVENILE IDIOPATHIC ARTHRITIS}

I. Nikishina ${ }^{1}$, S. Arsenyeva ${ }^{1}$, E. Fedorov ${ }^{1}$, V. Matkava ${ }^{2}$, A. Shapovalenko ${ }^{2}$, A. Panova ${ }^{3}$, E. Denisova ${ }^{4}{ }^{1}$ V. A. Nasonova Research Institute of Rheumatology, Paediatric, Moscow, Russian Federation; ${ }^{1}$ V. A. Nasonova Research Institute of Rheumatology, Paediatric, Moscow, Russian Federation; ${ }^{3}$ Moscow Scientific Research Institute of Ophthalmic Disease (Helmgoltz), Mosocw, Russian Federation; ${ }^{4}$ Moscow Scientific Research Institute of Ophthalmic Disease (Helmgoltz), Moscow, Russian Federation

Background: Juvenile Idiopathic Arthritis (JIA) is heterogeneous group of diseases which may include genetically determined conditions. Extremely rare monogenic hereditary autoinflammatory disease, such as Blau syndrome (BS) is usually difficult to recognize and JIA is initially established. BS is caused by a mutation in the NOD2/CARD5 gene and phenotypically characterized by triad of granulomatosis polyarthritis, uveitis and skin rash.

Objectives: To present the family case of genetically confirmed BS in 2 siblings, initially diagnosed as patients with JIA.

Methods: Two brothers of 15 and 3 years old were examined in our clinic. Additional genetic assay was performed because of unusual clinical picture.

Results: Case report. Older brother, 15 y.o. developed arthritis of both wrist joints at the age of two. There was erythematous maculo-micropapular scaly rash on the trunk and extremities before the onset of arthritis. By 2009 (5 y.o.), the knees, ankles, and three PIP joints of the left hand were involved. Polyarthritis was characterized by severe effusion and periarticular tissues swallowing. He was treated in regional hospital by NSAID, methotrexate, cyclosporine A without significant positive effect. Since 2012 etanercept was added for treatment with variable result. Inactive status of the disease is never reached. Repeated intra-articular GC injections were needed from 4 to 10 times per year. The patient was admitted to our clinic in November 2019. He was suffering from severe polyarthritis and uveitis de novo (granulomatous chorioretinitis) was detected. At the same time his younger $3 \mathrm{y}$. o. brother with recently started disease was hospitalized in our clinic. He had polyarthritis with typical features of "boggy" synovitis and tenosynovitis of wrists, ankles and knees, anterior uveitis was determined. The onset of arthritis was preceded by a small-spotted rash with desquamation. This classical clinical features in younger brother let us suggest the BS. There was no increasing of ESR and CRP in both sibs throughout the course of the disease. A molecular genetic study of the NOD2/CARD15 gene in both brothers revealed the same mutation of $c .1001 \mathrm{G}>A$ (p. Arg334GIn). Because of the absence of specific treatment for this disease and due to insufficient effect of etanercept with uveitis de novo therapy was changed to golimumab and good initial effect is reached.
The youngest brother has started his therapy by methotrexate. It should be noted that the family has the eldest brother (20 y.o.), who has been suffering from arthritis since an early childhood with similar clinical picture. We are going to perform genetic analysis of the NOD2/CARD15 gene for the eldest brother.

Conclusion: Our clinical case shows that extremely rare BS may be misdiagnosed as JIA. Lack of efficacy of the etanercept therapy and uveitis de novo developing may be caused by genetic (non-idiopathic) nature of disease. Classic triad of boggy-arthritis granulomatous uveitis and/or skin lesions without acute phase markers is required to perform genetic assay for the detection of a pathogenic mutation of the NOD2/CARD15 gene. This case is remarkable by the presence of BS in two (or 3) children of the family. References:

[1] Wouters C.H, Maes A, Foley K, et al. Blau Syndrome, the prototypic auto-inflammatory granulomatous disease. Pediatric Rheumatology. 2014; 12: 33.

Disclosure of Interests: None declared

DOI: 10.1136/annrheumdis-2020-eular.6239

\section{AB0997 \\ IS HEIGHT ADJUSTMENT NECESSARY IN PEDIATRIC DENSITOMETRY IN ALL CHILDREN?}

B. P. Magallares López ${ }^{1}, \underline{\text { H. Park }}{ }^{1}$, D. Cerda ${ }^{2}$, J. Betancourt $^{3}$, G. Fraga ${ }^{3}$, S. Boronat ${ }^{3}$, I. Gich ${ }^{4}$, A. M. Marín ${ }^{5}$, S. Herrera ${ }^{5}$, J. Malouf ${ }^{5}$, J. Casademont ${ }^{5}$, A. García-Guillén ${ }^{1}$, H. Corominas ${ }^{1} .{ }^{1}$ Hospital de la Santa Creu i Sant Pau, Rheumatology, Barcelona, Spain; ${ }^{2}$ Hospital de Sant Joan Despí Moisès Broggi, Rheumatology, Sant Joan Despí, Spain; ${ }^{3}$ Hospital de la Santa Creu i Sant Pau, Pediatrics, Barcelona, Spain; ${ }^{4}$ Hospital de la Santa Creu i Sant Pau, Epydemiology, Barcelona, Spain; ${ }^{5}$ Hospital de la Santa Creu i Sant Pau, Internal Medicine, Barcelona, Spain

Background: The current guidelines of the International Society for Clinical Densitometry (1) recommend that in children with linear growth or maturational delay, Z score results should be adjusted. Height for age $\mathrm{Z}$ score (HAZ) adjustment is valid and can be calculated using the formula the formula proposed by Zemmel et al(2). It is possible that pediatric populations without linear growth or maturational delay, also benefit from $\mathrm{HAZ}$, to prevent bone size from influencing the final $\mathrm{Z}$ score.

Objectives: To evaluate $Z$ score variability adjusted and without adjusting for height for age.

Methods: We analysed data from densitometry performed on patients 2-20 years of age, from 2016 to 2018 , assessed in the pediatric rheumatology office of our hospital for presenting risk factors for low bone mass/osteoporosis. The HAZ was calculated according to Zemel's formula.

Results: Data from 103 patients are presented. Its characteristics are summarized in Table 1

Table 1.

\begin{tabular}{ll}
\hline Mean age & 9,8 years \\
\hline Female & $52,4 \%$ \\
Height Percentil $\leq 3$ & $6,8 \%$ \\
Height Percentil $\geq 97$ & $4,9 \%$ \\
LBM (Z score $\leq-2)$ spine & $8,2 \%$ \\
LBM HAZ spine & $6,4 \%$ \\
LBM whole body & $10,5 \%$ \\
LBM HAZ whole body & $7,2 \%$
\end{tabular}

The table shows that the proportion of patients with BMD decreases in both the spine region and the whole body when adjusting for HAZ.

When evaluating the relationship between densitometric measurements we found that spine $Z$ score (ZsS) and whole body Z score (ZsWB) had a correlation coefficient of $0,73(p<0,001)$. There were no differences between their averages $(p=0,170)$.

At the LBM cut-off point ( $Z$ score $\leq-2)$ there were discrepancies in $7 \%$, where $5 \%$ presented LBM in ZsWB but not in ZsS. The concordance index at this point was 0,557 .

When comparing these measures with their HAZ adjusted equivalents, we observe:

HAZ adjusted ZsS vs ZsS without adjusting: There were no differences between their averages $(p=0,913)$ with a correlation coefficient of 0,78 $(p<0,001)$. Concordance index at cut-off point for LBM was 0,498 , with a discrepancy of $7 \%$, where $2 \%$ had LBM according to HAZ adjusted ZsS, but not to ZsS without adjusting

HAZ adjusted ZsWB vs ZsWB without adjusting: There were no differences between their averages $(p=0,367)$ with a correlation coefficient of 0,82 $(p<0,001)$. Concordance index at cut-off point for LBM was 0,557 , with a discrepancy of $7 \%$, where $2 \%$ had LBM according to HAZ adjusted ZsWB, but not to ZsWB without adjusting.

Conclusion: There are discrepancies at the LBM cut-off point depending on the HAZ adjustment.

The pediatric population without linear growth or maturational delay, can also benefit from HAZ adjustment, especially those with high height percentiles in which their size can hide a diagnosis of LBM. 
References:

[1] Weber DR, Boyce A, Gordon C, Hogler W, Kecskemethy HH, Misra M, et al. The Utility of DXA Assessment at the Forearm, Proximal Femur, and Lateral Distal Femur, and Vertebral Fracture Assessment in the Pediatric Population: 2019 ISCD Official Position. Journal of clinical densitometry: the official journal of the International Society for Clinical Densitometry. 2019;22(4):567-89

[2] Zemel BS, Leonard MB, Kelly A, Lappe JM, Gilsanz V, Oberfield S, et al. Height adjustment in assessing dual energy $x$-ray absorptiometry measurements of bone mass and density in children. The Journal of clinical endocrinology and metabolism. 2010;95(3):1265-73.

Disclosure of Interests: None declared

DOI: 10.1136/annrheumdis-2020-eular.861

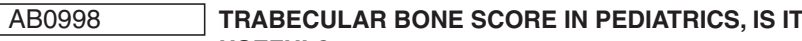 USEFUL?}

B. P. Magallares López ${ }^{1}$, H. Park ${ }^{* 1}$, D. Cerda ${ }^{2}$, J. Betancourt $^{3}$, G. Fraga $^{3}$ S. Boronat ${ }^{3}$, S. Herrera ${ }^{4}$, A. M. Marín ${ }^{4}$, I. Gich ${ }^{5}$, J. Malouf ${ }^{4}$, J. Casademont ${ }^{4}$ A. García-Guillén ${ }^{1}$, H. Corominas ${ }^{1} .{ }^{1}$ Hospital de la Santa Creu i Sant Pau, Rheumatology, Barcelona, Spain; ${ }^{2}$ Hospital de Sant Joan Despí Moisès Broggi, Rheumatology, Sant Joan Despí, Spain; ${ }^{3}$ Hospital de la Santa Creu i Sant Pau, Pediatrics, Barcelona, Spain; ${ }^{4}$ Hospital de la Santa Creu i Sant Pau, Internal Medicine, Barcelona, Spain; ${ }^{5}$ Hospital de la Santa Creu i Sant Pau, Epydemiology, Barcelona, Spain

Background: Bone fragility depends not only on bone mineral density (BMD), but also on bone microarchitecture. In adults, Trabecular Bone Score (TBS) is being used as an indirect marker of bone microarchitecture

It is a software that applicated to the vertebral image obtained by conventional densitometry, informs about the thickness of the trabeculae, the trabecular connectivity and the space between them. A high score indicates a better bone microstructure. In adults, a TBS equal to or greater than 1,350 is considered to represent a normal microarchitecture

Objectives: To evaluate the usefulness of TBS in pediatric population with risk factors for Low Bone Mass (LBM)

Methods: TBS was assessed by analyzing vertebral densitometries performed on patients from 4 to 20 years of age, assessed in the pediatric rheumatology office of our hospital for presenting risk factors for LBM, consecutively from 2016 until 2018 Data were compared with normal pediatric population

Results: Data from 83 patients are shown, with an average age of 11.2 years, $62 \%$ female, $80 \%$ Caucasian

The main risk factors for LBM were (\%): Insufficient calcium intake $(84,5)$, medications with osteopenizing potential (31), corticosteroids (39), sedentary lifestyle $(13,6)$, fractures of long or vertebral bones $(12,6)$ and hypovitaminosis $D(8,1)$

Table 1. TBS por age groups and in patients with and without LBM

\begin{tabular}{lcccc}
\hline Age groups & $\mathbf{n}$ & Mean & SD & Minimum-Maximum \\
\hline Scholars (4-9a) & 22 & 1,321 & 0,093 & $1,119-1,502$ \\
Adolescence (10-17a) & 54 & $\mathbf{1 , 3 0 9}$ & 0,088 & $1,073-1,493$ \\
Youth (18-20a) & 6 & 1,359 & 0,085 & $\begin{array}{c}1,258-1,460 \\
\text { Spine Z score }\end{array}$ \\
$\quad \mathbf{n}$ & Mean (SD) & $\mathrm{p}$ & Minimum-Maximum \\
$\quad>-2$ & 8 & $1,270(0,075)$ & 0,126 & $1,419-1,162$ \\
Whole Body Z score & 74 & $1,321(0,090)$ & & $1,502-1,073$ \\
$\quad \leq-2$ & 9 & $1,246(0,060)$ & $\mathbf{0 , 0 1 2}$ & $1,323-1,145$ \\
$>-2$ & 73 & $1,324(0,089)$ & & $1,502-1,073$ \\
\hline
\end{tabular}

Table 2. TBS in healthy population and study population for age

\begin{tabular}{lcccccc}
\hline & $\begin{array}{c}\text { Healthy girls } \\
(\mathrm{n}=2535)\end{array}$ & & $\begin{array}{c}\text { Healthy boys } \\
(\mathrm{n}=1459)\end{array}$ & & $\begin{array}{c}\text { Study girls } \\
(\mathrm{n}=47)\end{array}$ & $\begin{array}{c}\text { Study boys } \\
(\mathrm{n}=36)\end{array}$ \\
\hline Age $\mathbf{( y )}$ & Spine BMD & TBS & Spine BMD & TBS & TBS & TBS \\
\hline $\mathbf{1 - 2}$ & 0,40 & 1,325 & 0,37 & 1,272 & & \\
$\mathbf{2 - 3}$ & 0,51 & 1,363 & 0,46 & 1,267 & & 1,127 \\
$\mathbf{3 - 4}$ & 0,52 & 1,346 & 0,51 & 1,264 & 1,204 & \\
$\mathbf{4 - 5}$ & 0,60 & 1,346 & 0,60 & 1,267 & 1,237 & 1,243 \\
$\mathbf{5 - 6}$ & 0,60 & 1,288 & 0,56 & 1,269 & 1,330 & 1,368 \\
$\mathbf{6 - 7}$ & 0,65 & $\mathbf{1 , 2 8 0}$ & 0,60 & $\mathbf{1 , 2 3 2}$ & $\mathbf{1 , 3 1 8}$ & 1,422 \\
$\mathbf{7 - 8}$ & 0,67 & $\mathbf{1 , 2 6 8}$ & 0,64 & $\mathbf{1 , 2 4 4}$ & $\mathbf{1 , 3 3 9}$ & $\mathbf{1 , 3 4 5}$ \\
$\mathbf{8 - 9}$ & 0,71 & $\mathbf{1 , 2 6 6}$ & 0,68 & $\mathbf{1 , 2 2 8}$ & $\mathbf{1 , 2 4 4}$ & \\
$\mathbf{9 - 1 0}$ & 0,75 & $\mathbf{1 , 2 7 8}$ & 0,70 & $\mathbf{1 , 2 0 8}$ & $\mathbf{1 , 2 5 3}$ & $\mathbf{1 , 3 4 1}$ \\
$\mathbf{1 0 - 1 1}$ & 0,8 & $\mathbf{1 , 2 8 5}$ & 0,73 & $\mathbf{1 , 2 3 1}$ & $\mathbf{1 , 2 2 9}$ & $\mathbf{1 , 2 9 2}$ \\
$\mathbf{1 1 - 1 2}$ & 0,84 & $\mathbf{1 , 3 3 7}$ & 0,76 & $\mathbf{1 , 2 5 0}$ & $\mathbf{1 , 3 0 3}$ & $\mathbf{1 , 3 1 5}$ \\
$\mathbf{1 2 - 1 3}$ & 0,99 & $\mathbf{1 , 3 5 5}$ & 0,81 & $\mathbf{1 , 2 4 8}$ & 1,381 & $\mathbf{1 , 3 6 8}$ \\
$\mathbf{1 3 - 1 4}$ & 1,06 & $\mathbf{1 , 3 8 6}$ & 0,89 & $\mathbf{1 , 2 7 3}$ & $\mathbf{1 , 3 9 4}$ & $\mathbf{1 , 3 3 8}$ \\
$\mathbf{1 4 - 1 5}$ & 1,10 & 1,398 & 0,99 & $\mathbf{1 , 3 0 3}$ & 1,474 & $\mathbf{1 , 2 8 5}$
\end{tabular}

Table 2. TBS in healthy population and study population for age

\begin{tabular}{lcccccc}
\hline & $\begin{array}{c}\text { Healthy girls } \\
(\mathrm{n}=2535)\end{array}$ & & $\begin{array}{c}\text { Healthy boys } \\
(\mathrm{n}=1459)\end{array}$ & & $\begin{array}{c}\text { Study girls } \\
(\mathrm{n}=47)\end{array}$ & $\begin{array}{c}\text { Study boys } \\
(\mathrm{n}=36)\end{array}$ \\
\hline $\mathbf{A g e}(\mathrm{y})$ & Spine BMD & TBS & Spine BMD & TBS & TBS & TBS \\
\hline $\mathbf{1 5 - 1 6}$ & 1,14 & 1,405 & 1,08 & 1,311 & 1,368 & 1,406 \\
$\mathbf{1 6 - 1 7}$ & 1,17 & 1,405 & 1,15 & 1,334 & 1,332 & 1,371 \\
$\mathbf{1 7 - 1 8}$ & 1,17 & 1,404 & 1,20 & 1,328 & 1,374 & 1,285 \\
$\mathbf{1 8 - 1 9}$ & 1,17 & 1,404 & 1,16 & 1,314 & & \\
\hline
\end{tabular}

Conclusion: TBS was lower in the patients with LBM by whole body $Z$ score but not in those with LBM by spine $Z$ score. We observed a decrease in TBS in adolescence, not corresponding with a decrease in BMD, and that should not be interpreted as a pathological finding

Similar results have been described in other pediatric populations $(1,2)$, but larger studies are needed to evaluate this phenomenon. We hypothesize that it may be due to a higher rate of growth in adolescence, with a lower rate of calcium apposition into the osteoid material

References:

[1] Del Rio DS, Winthenrieth R. BONE MICROARCHITECTURE (TBS) AND BONE MASS DEVELOPMENT DURING CHILDHOOD AND ADOLESCENCE IN A SPANISH POPULATION GROUP. . WCO-IOF-ESCEO; Seville2014.

[2] Shawwa K, Arabi A, Nabulsi M, et al. Predictors of trabecular bone score in school children. Osteoporosis international: a journal established as result of cooperation between the European Foundation for Osteoporosis and the National Osteoporosis Foundation of the USA. 2016;27(2):703-10.

Disclosure of Interests: None declared

DOI: 10.1136/annrheumdis-2020-eular.866

\section{\begin{tabular}{|l|l}
\hline AB0999 HALLMARK CLINICAL FEATURES OF JUVENILE \\
\hline
\end{tabular} PSORIATIC ARTHRITIS (JPSA)}

K. Aleksanyan ${ }^{1}$, S. Chebysheva ${ }^{1}$, A. Meleshkina ${ }^{1}$, E. Popova ${ }^{1}$, E. Zholobova ${ }^{1}$. ${ }^{1}$ I.M. Sechenov First Moscow State Medical University, Moscow, Russian Federation

Background: JPsA is presented in 4 to $9 \%$ children with juvenile arthritis $[1,2]$ According to ILAR criteria, patients with uveitis, HLA-B27-positivity, and other exclusion criteria cannot be included in the JPsA category. Therefore, in pediatric rheumatological practice, the Vancouver criteria are more often used [3]

Objectives: to identify clinical features of psoriatic arthritis in children.

Methods: a single center, prospective and retrospective, continuous clinical trial for the period from 1989 to 2019 was conducted.

Results: We observed 83 patients with JPsA aged from 4 months to 17 years Fifty-nine (71\%) patients had definite JPsA and $24(29 \%)$ had probable JPsA according to the Vancouver criteria. The clinical and demographic characteristics are presented in Table 1.

In $17(29 \%)$ children with definite JPsA, skin lesions presented as the firs sign of the disease, joint damage in these patients developed after $3.5 \pm 2$ (2) years. In 28 (48\%) patients articular syndrome was observed on the onse with subsequent skin manifestations after $5.3 \pm 3(5)$ years. Fourteen $(23 \%)$ children with definite JPsA had simultaneous debut of skin and articular syndromes (Fig.1)

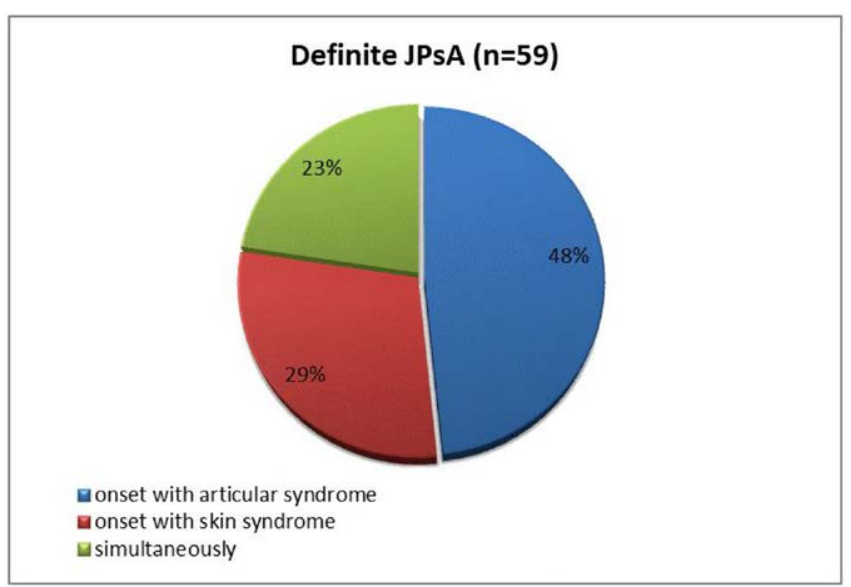

Fig.1. Skin and / or articular syndromes in the onset of the disease in children with definite JPsA 